\title{
RESISTÊNCIA AO IMPACTO CHARPY E IZOD EM COMPOSITOS DE MATRIZ DE EPÓXI REFORÇADOS COM FIBRAS DE FIQUE*
}

\section{Resumo}

Maria Carolina Andrade Teles ${ }^{1}$ Marcos Vinícius Fonseca Ferreira ${ }^{2}$ Felipe Perissé Duarte Lopes ${ }^{3}$ Frederico Muylaert Margem ${ }^{4}$ Sergio Neves Monteiro ${ }^{5}$ Luís Carlos da Silva ${ }^{6}$

Vários motivos levaram a priorizarem o uso de fibras naturais em detrimento do uso de fibras sintéticas, sobretudo as razões sociais e ambientais. A fibra deste trabalho é extraída da folha da planta - Furcraea Andina (da Colômbia, onde usam esta fibra para a produção de artesanatos e sacos) e demonstra um grande potencial como reforço de compósitos poliméricos. Este trabalho avaliará o teste de impacto de Izod e Charpy de compósitos com matriz epóxi reforçada com diferentes porcentagens de fibras. Para os testes, as fibras de fique foram misturadas com à resina epóxi em um molde metálico retangular, o conjunto foi submetido a uma pressão e sofreram um processo de cura à temperatura ambiente durante 24 horas. As amostras produzidas foram submetidas aos ensaios seguindo a norma ASTM D256 (Método de Ensaio Padrão para à Determinação da Resistência ao Impacto do Pêndulo Izod para Plásticos) e a norma ASTM D6110 (Método de Ensaio Padrão para Determinar a Resistência ao Impacto Charpy para Plásticos). Os compósitos continham até $30 \%$ em volume de fibras de fique, que foram alinhadas ao longo de todo o comprimento da matriz. As amostras submetidas à ambos os ensaios (Izod e Charpy) tiveram suas fraturas analisadas por microscopia eletrônica de varredura (MEV). Houve um aumento significativo da resistência ao impacto de acordo com o aumento da porcentagem volumétrica das fibras de fique. Esse desempenho pode estar associado ao mecanismo de fratura gerado a partir da adesão entre as fibra de fique e a matriz colaborando na absorção da energia de impacto, além do fato de que a fibra é capaz de dificultar a ruptura das amostras.

Palavras-chave: Fibras de fique; Impacto Izod; Impacto Charpy; Resina epóxi; Compósitos.

\section{CHARPY AND IZOD IMPACT RESISTENCE IN EPOXY MATRIX COMPOSITES REINFORCED WITH FIQUE FIBERS}

\section{Abstract}

Several issues have maximized the permutation between the use of synthetic fibers for the use of natural fibers, above all for social and environmental reasons. The fiber used is extracted from the leaf of the plant - Furcraea Andina (from Colombia, where they use fiber for the production of handicrafts and sacs), demonstrates a great potential as reinforcement of polymeric composite. This work will evaluate impact test of composites with epoxy matrix associated to different percentages of fibers, both Izod and Charpy configurations. For the tests, the fibers were mixed with the DGEBA / TETA epoxy resin and fique fibers under pressure in a rectangular metal mold, cured at room temperature for 24 hours. The specimens generated followed the ASTM D256 (Standard Test Methods for Determining the Izod Pendulum Impact Resistance of Plastics) and ASTM D6110 (Standard Test Method for Determining the Charpy Impact Resistance of Notched Specimens of Plastics), were up to $30 \%$ by volume of fique fibers, which were aligned along the entire matrix length. The specimens tested for both tests (Izod and Charpy) had their fractures analyzed by Scanning Electron 
Microscopy (SEM). Obtaining a significant increase in impact strength according to the volumetric percentage fibrous growth that reinforced the composite. Such performance may be associated with the fracture mechanism generated from the interaction between the fique fiber and matrix - it has helped in the absorption of impact energy, in addition to the fact that the fiber is able to hamper the rupture of specimens.

Keywords: Fique fibers, Impact izod, Impact charpy, epoxy resin, composites.

1 Engenheiro Civil, M.sc., Mestre em Ciência dos Materiais, Seção de Engenharia Mecânica e de Materiais, Instituto Militar de Engenharia, Rio de Janeiro, RJ Brasil.

2 Engenheiro Civil, M.sc., Mestre em Ciência dos Materiais, Seção de Engenharia Mecânica e de Materiais, Instituto Militar de Engenharia, Rio de Janeiro, RJ Brasil.

3 Engenheiro Civil, M.sc., Mestre em Ciência dos Materiais, Seção de Engenharia Mecânica e de Materiais, Instituto Militar de Engenharia, Rio de Janeiro, RJ Brasil.

4 Engenheiro Civil, M.sc., Mestre em Ciência dos Materiais, Seção de Engenharia Mecânica e de Materiais, Instituto Militar de Engenharia, Rio de Janeiro, RJ Brasil.

5 Engenheiro Metalurgista, Ph.D, Professor Titular, Seção de Engenharia Mecânica e de Materiais, Instituto Militar de Engenharia, Rio de Janeiro, RJ, Brasil.

6 Engenheiro Químico, D.Sc., Doutor em Ciência dos Materiais, Seção de Engenharia Mecânica e de Materiais, Instituto Militar de Engenharia, Rio de Janeiro, RJ, Brasil. 


\section{INTRODUÇÃO}

Muito foi dito sobre à exploração e uso de recursos naturais na indústria e como eles podem substituir os produtos sintéticos já utilizados no mercado industrial. $\mathrm{O}$ fato de serem originários de recursos renováveis e produzirem uma quantidade relativamente pequena de poluentes, favorecem o seu uso se forem comparados com alguns materiais reforçados com sintéticos. Um exemplo diretamente ligado a esta tendência substitutiva são as fibras de vidro, que estão sendo substituidas por fibras naturais [18]. As fibras lignocelulósicas são obtidas à partir de recursos renováveis e o seu processamento produzem baixas emissões de $\mathrm{CO}_{2}$, além de serem recicláveis e biodegradáveis. Um outro fator apreciável é que as fibras naturais precisam de energia relativamente menor para serem processadas em comparação com as fibras sintéticas que contribuem diretamente para o aquecimento global. Economicamente, as fibras naturais são significativamente mais baratas do que as fibras de vidro, mesmo estas sendo as mais baratas das fibras sintéticas [9-10]. As propriedades mecânicas obtidas a partir dos ensaios com fibras naturais atingem, em alguns casos, valores competitivos comparados com os resultados apresentados pelas fibras de vidro [11-14]. Sabe-se que os compósitos poliméricos reforçados com fibras naturais, longas e alinhadas apresentam um melhor desempenho das suas propriedades mecânicas quando comparadas a outras formas de disposição na matriz polimérica (como fibras curtas ou fibras orientadas de forma aleatória). Trabalhos anteriores analisaram o desempenho das fibras de fique, avaliando através do ensaio de impacto de Charpy e o ensaio de impacto de Izod, desta fibra incorporada à matriz de resina epóxi e os resultados obtidos foram comparados [15-16].

\section{MATERIAIS E MÉTODOS}

Os materiais utilizados neste trabalho foram as fibras naturais extraídas da folha da planta de fique (FIGURA 1), que foi fornecida por produtores da Colômbia. Não foram aplicados tratamentos na superfície das fibras.

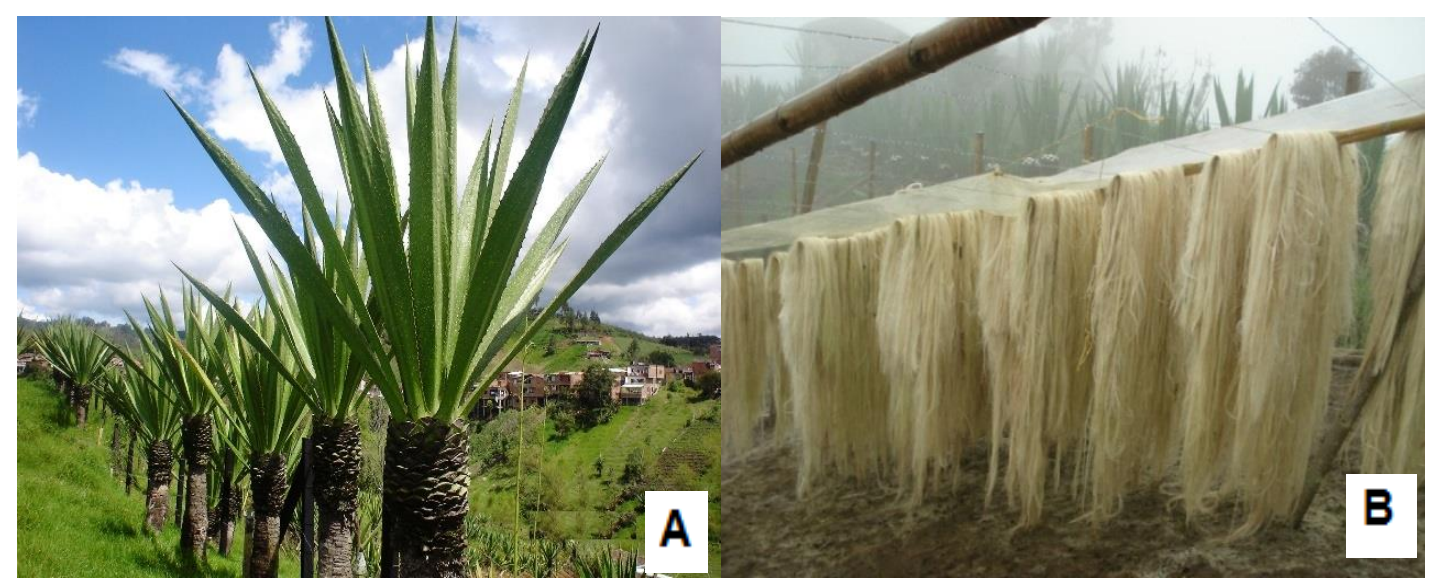

Figura 1. Plantações de fique $(A)$ e fibras de fique $(B)$

As fibras de fique, com quantidade variando de 0 a $30 \%$ em volume, foram alinhadas em uma matriz de metal retangular com dimensões : 12,7 x $125 \times 152 \mathrm{~mm}$ e misturadas com a resina epóxi (DGEBA) já misturada com o endurecedor com $0,5 \%$ de catalisador à base de metil-etil-cetona (TETA). Os compósitos foram colocados para curar submetidos à uma pressão de 5 toneladas à temperatura ambiente durante 24 horas. Essas placas foram utilizadas na confecção de amostras com formatos 
adequados para os ensaios de impacto Izod e Charpy de acordo com as normas ASTM D256 e ASTM D6110 [17-18], respectivamente. As amostras foram submetidas ao impacto de um pêndulo PANTEC, modelo XC - 50 com configurações Izod e Charpy. A energia de impacto foi obtida usando um martelo de $11 \mathrm{~J}$ para as composições que variam de 0 a 30\%. Foram utilizadas 12 amostras de cada composição e os resultados foram analisados estatisticamente.

\section{RESULTADOS E DISCUSSÃO}

A TABELA 1 mostra os resultados da energia de impacto Izod com seus respectivos desvios padrão para epóxi puro e para compósitos reforçados com diferentes frações volumétricas de fibras de fique.

TABELA 1. Energia de impacto Izod para compósitos de matriz epóxi reforçadas com fibras de fique

\begin{tabular}{c|c}
\hline Quantidade de fibra (\%) & Energia de Impacto $(\mathrm{J} / \mathrm{m})$ \\
\hline 0 & $13.00 \pm 02.00$ \\
\hline 10 & $165.70 \pm 29.70$ \\
\hline 20 & $231.10 \pm 35.30$ \\
\hline 30 & $303.91 \pm 42.50$ \\
\hline
\end{tabular}

Pode-se observar pelos dados da TABELA 1, que uma fração de volume de fibra de $30 \%$ aumenta a energia de impacto Izod em mais de vinte vezes, revelando que as fibras de fique são um bom material de reforço para a resistência ao impacto. Esse fenômeno acontece, porque as fibras agem como uma barreira à propagação de trincas, desviando a propagação e exigindo uma quantidade de energia 20 vezes maior do que a energia necessária para fraturar uma matriz de epóxi sem reforço. De acordo com Yue et al. (1995) este comportamento está de acordo com o mecanismo de fratura de compósitos de baixa adesão [19]. O gráfico da energia absorvida no ensaio de impacto de Izod versus à fração volumétrica correspondente ao compósito é demonstrado na FIGURA 2. Esta figura mostra um aumento significativo na energia de impacto de Izod com o aumento na quantidade de fibras de fique. 


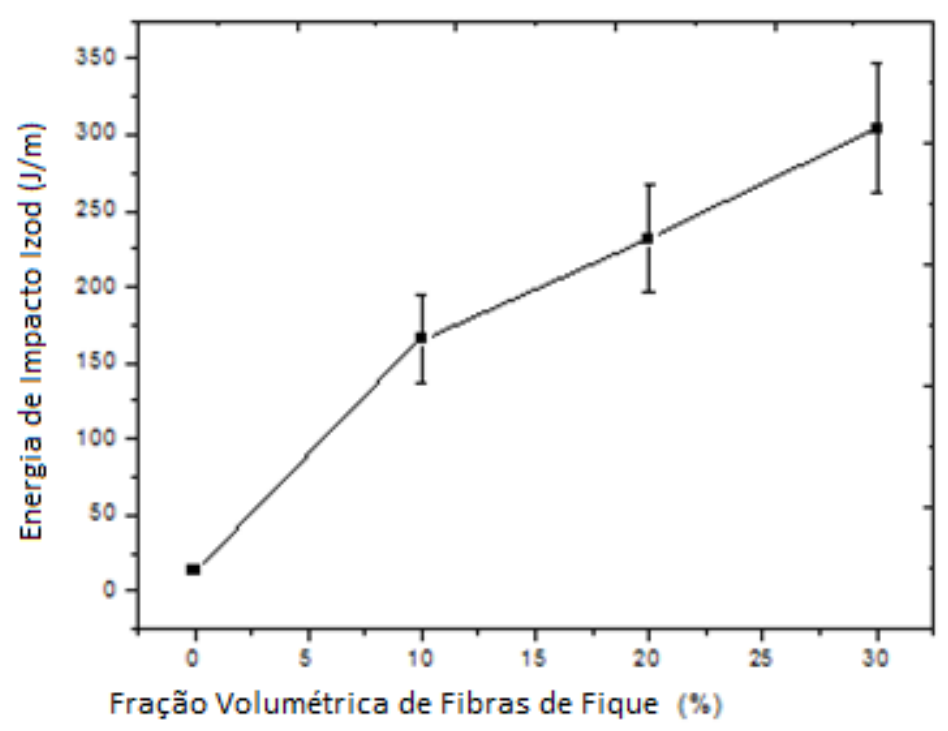

FIGURA 2. Energia de Impacto Izod com a quantidade de fibras de fique no compósito

A FIGURA 2 indica um aumento na resistência ao impacto com o aumento do volume de fibras de fique. Esse aumento é devido à uma maior área de fratura produzida pela propagação longitudinal da trinca que ocorre entre a matriz e as fibras, esse comportamento também é considerado comum em compósitos de baixa adesão [19]. A FIGURA 3 mostra macroscopicamente, um aumento na tenacidade do compósito com o aumento da quantidade de fibras.

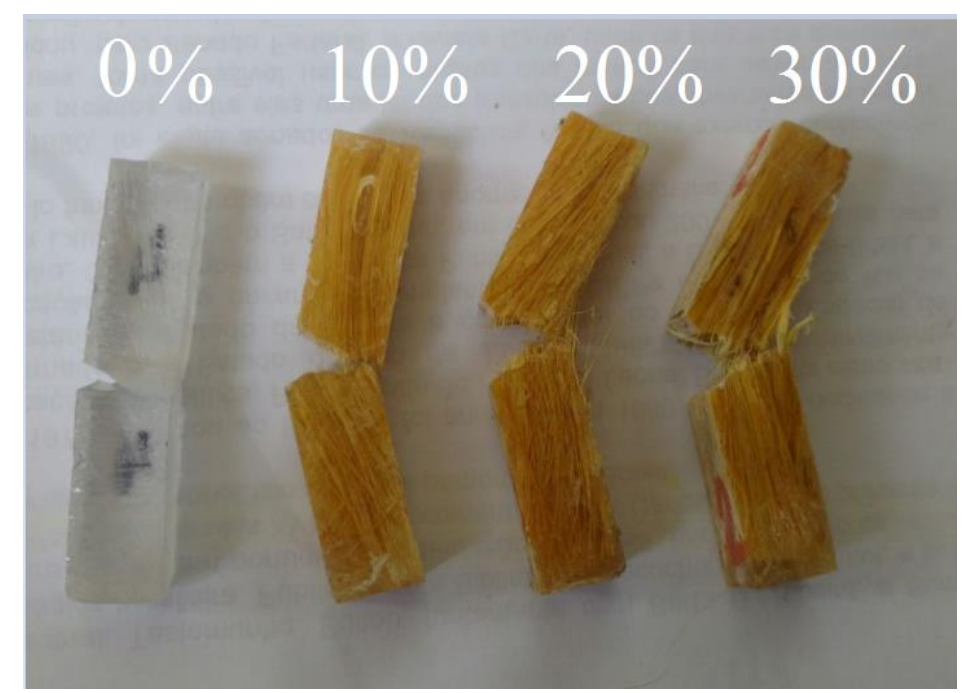

FIGURA 3. Macroestrutura de compósitos fraturados após os ensaios de Izod com diferentes quantidades de fibras

A TABELA 2 apresenta os resultados dos ensaios de impacto Charpy para compósitos de matriz epóxi reforçados com diferentes frações volumétricas de fibras de fique. 
TABELA 2. Energia de impacto Charpy para compósitos de matriz epóxi reforçadas com fibras de fique

\begin{tabular}{c|c}
\hline Quantidade de fibra $(\%)$ & Energia de Impacto $(\mathrm{J} / \mathrm{m})$ \\
\hline 0 & $151.39 \pm 22.51$ \\
\hline 10 & $241.70 \pm 75.42$ \\
\hline 20 & $365.92 \pm 78.37$ \\
\hline 30 & $440.76 \pm 42.14$ \\
\hline
\end{tabular}

Com base nos resultados mostrados na TABELA 2, a FIGURA 4 demonstra a relação da energia de Charpy com a quantidade de fibras de fique reforçando o compósito.

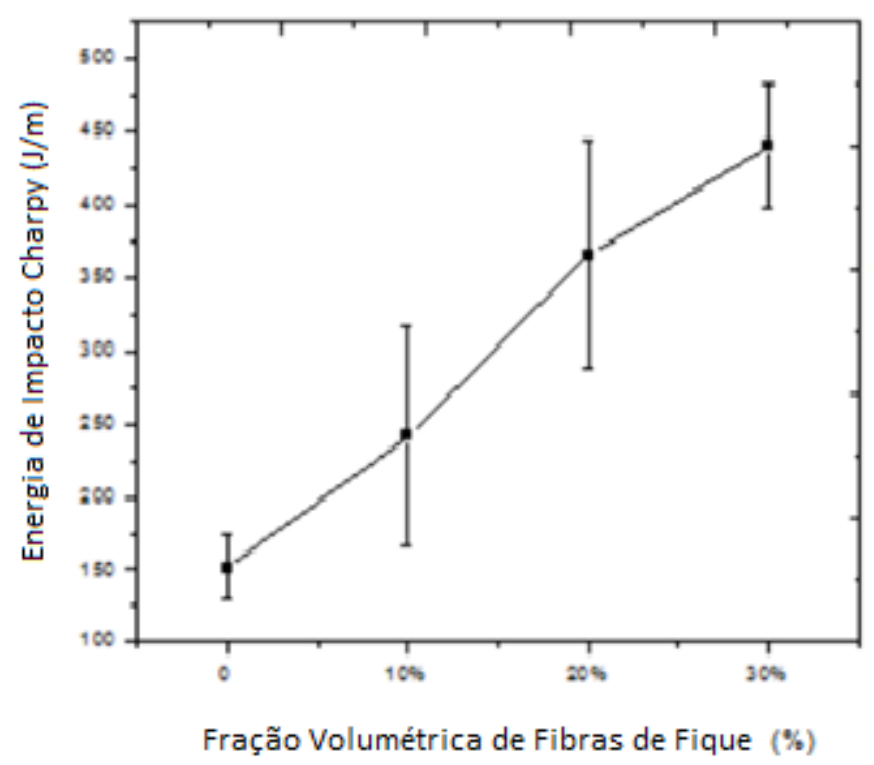

FIGURA 4. Energia de Impacto Charpy com a quantidade de fibras de fique no compósito

\section{$0 \%$

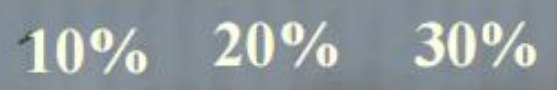

FIGURA 5. Macroestrutura de compósitos fraturados após os ensaios de Charpy com diferentes quantidades de fibras 
Para porcentagens de fibra de 10, 20 e 30\%, os compósitos não foram completamente fraturados após o impacto. Este fenômeno acontece devido à alta resistência à tração da fibra de fique, evitando assim um colapso total. É importante notar que o crescimento de volume da fibra aumenta à resistência das amostras, uma vez que não há ruptura com altos volumes de fibras, indicando que a energia necessária para a fratura das amostras é maior.

\section{CONCLUSÃo}

Compósitos reforçados com fibras de fique possuem uma resistência ao impacto maior que a resina pura, demonstrando que as fibras de fique são um bom material de reforço para compósitos. A resistência ao impacto aumenta linearmente com a incorporação de fibras de fique em até $30 \%$ em volume. Algumas fibras de fique ficaram íntegras após os ensaios de impacto, este fenômeno é devido à baixa adesão das fibras a matriz, resultando em maior energia de impacto absorvida por causa da propagação de trincas pela interface fibra / matriz, permitindo a formação de uma maior área de fratura longitudinal em relação a uma fratura transversal que ocorre no epóxi puro.

\section{REFERÊNCIAS}

1 Mohanty AK, Misra M, Drzal LT. Natural Fibers, Biopolymers and Biocomposites, Taylor \& Francis, Bouca Raton - FL, 2005.

2 Neto FL and Pardini LC. Compósitos Estruturais, Edgard Blücher, São Paulo SP, 2006.

3 Monteiro SN, Lopes FPD, Ferreira AS and Nascimento DCO. Natural fiber polymer matrix composites: cheaper, tougher and environmentally friendly. JOM, 61(1); 2009. P. 17-22.

4 Satyanarayana KG, Sukumaran K, Kulkarni AG, Pillai SGK, Rohatgi PK. Fabrication and properties of natural fiber-reinforced polyester composites. Composites; 1999. V. 17, p. 329-274.

5 Crocker J. Natural materials innovative natural composites. Materials Technology, 2-3; 2008. P. 174-178.

6 Callister WD. Materials Science and Engineering - An Introduction 7ed., Jhon Wiley \& Sons, New York - NY, 2007.

7 Mallick CK. Fiber-reinforced Composites - Materials, Manufacturing and Design, LLC, Boca Raton - FL, 2007.

8 Lucas AA, Ambrósio JD, Bonse BC, Bettini SHC. Natural Fiber Polymer Composites Technology Applied to the Recovery and Protection of Tropical Forests Allied to the Recycling of Industrial and Urban Residues, Advances in Composite Materials - Analysis of Natural and Man-Made Materials, Intech, p. 165 - 194, 2011.

9 Satyanarayana KG, Guimarães JL and Wypych F. Composites Part A Vol. 38 (2007), p. 1694

10 Gore A. An Inconvenient Truth. The Planetary Emergency of Global Warming and What We Can Do About It. (Emmaus, Pennsylvania, USA: Rodale Press, 2006).

11 Wambua P, Ivens I, Verpoest I. Natural fibers: can they repalce glass and fibre reinforced plastics?, Composites Science and Technology, 63 (2003) 12591264. 
12 Mohanty AK, Misra M and Hinrichsen G. Biofibers, biodegradable polymers and biocomposites: an overview, Macromolecular Mat. And Engineering, 276/277 (2000), 1-24.

13 Monteiro SN, Ferreira AS and Lopes FPD. Global Symposium on Recycling, Waste Treatment and Clean Technology (REWAS). Cancun 12-15 October 2008. Proceeding...Cancun 2008. (Mexico). p.1653.

14 Leão AL, Tan IH and Caraschi, JC. International Conference on Advanced Composites. Hurghada 1998. Egypt. p. 557.

15 Giacomini NP. Compósitos reforçados com fibras naturais para a indústria automobilística, Dissertação de Mestrado, USP - Universidade de São Paulo, São Carlos-SP, p. 21, 2003.

16 Altoe GR, Margem FM and Monteiro SN. 67ํㅡㄹ Congresso Internacional da Associação Brasileira de Metalurgia, Materiais e Mineração (ABM) Rio de Janeiro 31 de Julho - 03 de Agosto 2012. p 55.

17 ASTM - American Society for Testing Materials. ASTM D256 - 02 - Standard Test Methods for Determining the Izod Pendulum Impact Resistance of Plastics. Annual Book of ASTM, 2002.

18 ASTM - American Society for Testing Materials. ASTM D6110 - 04 - Standard Test Methods for Determining the Charpy Pendulum Impact Resistance of Notched Specimens of Plastics. Annual Book of ASTM, 2004.

19 Yue CY, Looi HC, Quel MY. Assessment of Fibre-Matrix Adhesion and Interfacial Properties Using the Pullout Test. Int. J. Adhesion and Adhesives, v. 15, p. $73-80,1995$. 